\title{
Anemia hemolítica autoinmune: una rara complicación de la colitis ulcerosa
}

\author{
M. VALDERRAMA ROJAS, F. J. RODRÍGUEZ GOROSTIZA, L. A. ÁLVAREZ-SALA \\ WALTHER, F. J. TORRES SEGOVIA, J. MILLÁN NÚÑEZ-CORTÉS \\ Servicio de Medicina Interna III. Hospital General Universitario Gregorio Marañon. \\ Madrid
}

AUTOIMMUNE ANAEMIA HAEMOLYTIC: A RARE COMPLICATION OF ULCERATIVE COLITIS

\begin{abstract}
RESUMEN
La anemia constituye un hallazgo frecuente en los pacientes con colitis ulcerosa, y su etiología suele ser multifactorial. Puede deberse a un sangrado crónico, a déficits nutricionales, a la toma de determinada medicación como la salazopirina, o bien tratarse de una anemia relacionada con una enfermedad de cáracter crónico como viene a serlo la colitis ulcerosa. Sin embargo, esta enfermedad se asocia frecuentemente a diversos trastornos inmunológicos, entre ellos, la anemia hemolítica autoinmune. Esta complicación, aunque rara, puede hallarse en el 0,2 al $0,7 \%$ de los pacientes afectos de colitis ulcerosa, y hasta en un $1,82 \%$ de los pacientes con esta enfermedad pueden presentar un test de Coombs directo positivo sin evidencia de hemolisis. Presentamos un nuevo caso de un paciente con colitis ulcerosa y anemia hemolítica autoinmune.
\end{abstract}

PALABRAS CLAVE: Colitis ulcerosa. Anemia hemolítica autoinmune.

\begin{abstract}
Anaemia is a common problem in pacients with ulcerative colitis, and its etiology is usually multifactorial. It can be produced by chronic blood loss, nutritional deficiencies, drugs such as salazopyrine, or it can be related to those chronic disease. However, ulcerative colitis is kno wed to be associated with several immune disorders, as autoimmune haemolytic anaemia. Nevertheless, this rare complication can be found in 0.2 to $0.7 \%$ of patients affected by ulcerative colitis, and although $1.82 \%$ of patients with ulcerative colitis have a positive direct Coombs test without evidence of hemolysis. We report one new case of ulcerative colitis associated with autoimmune anaemia haemolytic, and a review the literature.
\end{abstract}

KEY WORDS: Ulcerative colitis. Autoimmune haemolytic anaemia.

Valderrama Rojas M, Rodriguez Gorostiza FJ, Álvarez-Sala Walther LA, Torres Segovia FJ, Millán Nuñez-Cortés J. Anemia hemolitica autoinmune: una rara complicación de la colitis ulcerosa. An Med Interna (Madrid) 2003; 20: 78-80.

\section{INTRODUCCIÓN}

La primera descripción de una anemía hemolítica autoinmune (AHAI) en el seno de una colitis ulcerosa (CU), fue realizada en 1955 por Lorber (4). En 1964, Edwards encontró un nuevo caso entre 624 pacientes con el diagnóstico de CU (5). En 1967, Ormerod presentó otro caso entre 479 pacientes (6). En un estudio prospectivo realizado en Portugal en 1991 y llevado a cabo en 180 pacientes, se concluyó que la frecuencia de esta complicación era del 0,6\% (7). En una serie de Oxford de 1989 con 858 pacientes se encontraron 4 casos asociados a AHAI (8). En la serie más amplia publicada hasta el momento, y llevada a cabo en el Hospital Mount Sinai en 1989, con un total de 1150 pacientes se encontró una prevalencia de AHAI del 0,7\% (2). En otro estudio la prevalencia del test de
Coombs positivo en pacientes con CU fue del $1,82 \%$, incluyen aquellos casos con o sin manifestaciones clínicas de hemolisis (3). Por último, en una serie publicada en 1997 con 302 pacientes con enfermedad ulcerosa se halló una prevalencia de AHAI en el $1,7 \%$ de los casos. Por lo tanto, hasta la actualidad se han descrito unos 50 casos de este trastorno inmunológico como complicación de una CU (9).

\section{CASO APORTADO}

Paciente varón de 70 años, sin antecedentes alérgicos medicamentosos, diabético con control dietético, y diagnosticado de colitis ulcerosa de 10 años de evolución con ocasionales episodios de anemización en relación con anemia ferropénica secundaria a sangrado digestivo. En el momento de su ingreso se encontraba en trata-

Trabajo aceptado: 19 de Noviembre de 2001

Correspondencia: Mónica Valderrama Rojas. Servicio de Medicina Interna III. Hospital General Universitario Gregorio Marañón. C/ Dr. Esquerdo, 46. 28007 Madrid. 
miento con Lixacol y Metronidazol. Acudió a Urgencia por astenia progresiva de 2 meses de evolución, sin alteraciones del ritmo intestinal (3-4 deposiciones al día) ni rectorragías ni dolor abdominal. A la exploración se objetivó discreta palidez cutánea, una auscultación cardiopulmonar rítmica a 80 l.p.m. con murmullo vesicular conservado, el abdomen era blando, no doloroso sin masas ni megalias con ruidos hidroaéreos conservados. Entre las pruebas complementarias destacaba en el hemograma: hematíes 2.200.000, hemoglobina 6,7 g/dl, hematocrito 19\%, VCM 80 fl, HCM 25,8 pg, leucocitos 7.400 con fórmula normal, plaquetas 413.000 ; en la bioquímica: glucosa $283 \mathrm{~g} / \mathrm{dl}$, urea $42 \mathrm{mg} / \mathrm{dl}$, creatinina $1 \mathrm{mg} / \mathrm{dl}$, LDH 409 U/l, GOT 17 U/l, GPT $6 \mathrm{U} / 1$, fosfatasa alcalina $188 \mathrm{U} / 1$, GGT $8 \mathrm{U} / 1$, proteínas totales 7,3 g/dl, Na $133 \mathrm{mmol} / \mathrm{l}, \mathrm{K} 3,4 \mathrm{mmol} / \mathrm{l}$; y en la coagulación: fibrinógeno $474 \mathrm{mg} / \mathrm{dl}$; actividad de protrombina 99\% INR 1, APTT 31 sg. En la radiografia de toráx se objetivó una discreta escoliosis dorsal y calcificación parietal aórtica, y la de abdomen, un patrón gaseoso inespecífico con aparente pérdida de haustración en colon, y osteopenia radiológica. Ante los hallazgos encontrados se llevó a cabo la transfusión de dos concentrados de hematíes, alcanzando un nivel de hemoglobina de $11,8 \mathrm{~g} / \mathrm{dl}$. Al $2^{\circ}$ día de su ingreso el paciente presentó, en un control analítico, una hemoglobina de 9,4 g/dl, siendo de $7,5 \mathrm{~g} / \mathrm{dl}$ al cabo de 5 días. Se inició estudio de anemia objetivándose: VSG $112 \mathrm{~mm}$; frotis sanguíneo con intensa anisocitosis, policromatofilia, macrocitos, punteado basófilo, esferocitos, y plaquetas grandes; bilirrubina total $2,7 \mathrm{mg} / \mathrm{dl}$, bilirrubina directa $0,8 \mathrm{mg} / \mathrm{dl}, \mathrm{LDH}$ $581 \mathrm{U} / \mathrm{l}$, hierro $69 \mathrm{ug} / \mathrm{dl}$, IST 24\%, transferrina $182 \mathrm{mg} / \mathrm{dl}$, haptoglobina $<7,63 \mathrm{mg} / \mathrm{dl}$, ferritina $226 \mathrm{ug} / \mathrm{l}$, vitamina B 12474 pg/ml, ácido fólico 5,6 ng/ml; Coombs directo positivo con especificidad Anti e (Coombs directo monoespecífico Ig G positivo, C3 negativo e Ig M negativo); hormonas tiroideas normales; CEA 2,6 ng/ml; PCR 3,46 $\mathrm{mg} / \mathrm{dl}(0-0,8)$; hemorragia oculta en heces positiva, y coprocultivo, parásitos, clostridium difficile y BAAR negativos. La ecografía abdominal mostraba un hígado de tamaño normal con discreta infiltración grasa, litiasis de vesícula biliar, y granuloma en zona inferior del bazo. En la colonospia se objetivó la presencia de hemorroides externas, e inflamación de colon moderada-severa compatible con colitis ulcerosa. En el estudio histológico se observó la presencia de colitis ulcerosa crónica en fase activa con zonas de actividad severa. Ante la existencia de anemia hemolítica y el grado de actividad de su proceso intestinal se pautó tratamiento con esteroides, con buena respuesta. El paciente fue dado de alta (con $\mathrm{Hb} 8,7 \mathrm{~g} / \mathrm{dl}$ ) y acudió a revisión al cabo de un mes objetivándose en un hemograma de control una hemoglobina de $11 \mathrm{~g} / \mathrm{dl}$, sin datos analíticos de hemolisis.

\section{DISCUSIÓN}

La anemia constituye un hallazgo frecuente en la $\mathrm{CU}$, en general asociada a un sangrado agudo o crónico por vía digestiva y una dificultad en la liberación de hierro de los depósitos orgánicos de ferritina en relación con cualquier proceso inflamatorio crónico. Sin embargo, la anemia también puede ser consecuencia del tratamiento, yq que la sulfasalazina puede producir anemia megaloblástica al impedir la absorción de folatos o ser causa de una hemólisis en pacientes con déficit de glucosa-6-fosfato-deshidrogenasa eritrocitaria. Otras formas de anemia hemolítica asociadas a la CU es la microangiopática, ocasionalmente asociada al síndrome hemolítico-urémico $(10,11)$. En el caso que presentamos no existían datos clínicos que hiciesen sospechar la presencia de una enfermedad infecciosa intermitente como causa de AHAI $(12,13)$. La transfusión de hematíes recibida por el paciente podría inducir a pensar en una hemólisis intravascular postransfusional, sin embargo, esta posibilidad quedó totalmente descartado con la presencia de un test de Coombs directo positivo con anticuerpos tipo IgG con especificidad anti e.

La etiopatogenia de la AHAI en la colitis ulcerosa aún no ha sido aclarada. Se ha especulado sin éxito sobre la existencia de una absorción inadecuada de sustancias antigénicas (haptenos) a través del intestino enfermo, que presentarían una reacción cruzada con los antígenos de superficie de los hematíes (14). Otra teoría afirmaba una posible reacción cruzada entre antígeno de la mucosa del colon y de los hematíes (15). Un estudio más reciente considera el colon como una fuente de autoanticuerpos este estudio fue realizado con pacientes afectos de CU y AHAI se demostró la presencia de anticuerpos IgG anti - eritrocito en las células mononucleares extraídas del colon de estos sujetos. Sin embargo, esta producción no pudo demostrarse en el cultivo in vitro de las células mononucleares extraídas de ganglios linfáticos, bazo y sangre periférica (16).

También se ha postulado una posible predisposición genética como desencadenante de una respuesta inmunológica aberrante en determinados pacientes con enfermedad inflamatoria intestinal, basado en el hallazgo de una prevalencia tres veces mayor de trastornos autoinmune en este grupo de pacientes que en la población general (17).

La AHAI parece darse más frecuentemente en mujeres, sin predilección por un grupo de edad. Puede preceder, acompañar o seguir al diagnóstico de CU, siendo el intervalo de tiempo establecido entre su disgnóstico y la aparición de la AHAI de unos 17 meses (9). Aunque no exista unanimidad, se describe una relación directa entre el grado de actividad de la enfermedad ulcerosa y la aparición de la AHAI (18). Así el $80 \%$ de los pacientes con $\mathrm{CU}$ y un test de Coombs directo positivo presentan una severa actividad de su proceso ulceroso.

El tratamiento correcto de la AHAI en la CU consiste en el empleo de esteroides a altas dosis. Cuando la respuesta al tratamiento no haya sido concluyente puede plantearse la incorporación de un fármaco inmunosupresor. La respuesta es a menudo poco satisfactoria, siendo necesaria la práctica de una colectomia total con o sin esplenectomia para el control de la hemólisis (2).

\section{Bibliografía}

1. Ramakrishna R, Manoharan A. Auto-immune haemolytic anaemia in ulcerative colitis. Acta Haemtol 1994; 91: 99-102.

2. Gumaste V, Greenstein AJ, Meyers R et al. Coombs-positive autoimmune hemolytic anemia in ulcerative colitis. Dig Dis Sci 1989; 34: 1457-61.

3. Poulsen LO, Feund L, Lylloff K et al. Positive Coombs test associated with ulcerative colitis. A prevalence study. Acta Med Scand 1988; 223: 75-8.

4. Lorber M, Schwartz LI, Wasserman LR. Association of antibody-coated red blood cells with ulcerative colitis. Am J Med 1955; 19: 887-96.

5. Edwards F, Truelove SC. The course and prognosis of ulcerative colitis. Gut, 1964; 5: 1-22.

6. Ormerod TP. Observations on the incidence and cause of anaemia in ulcerative colitis. Gut 1967; 8: 107-14. 
7. Tavarela VF, Fraga J, Carvalho J, et al. Autoimmune hemolytic anemia in ulcerative colitis: A case report with review of the literature. J Clin Gastroenterol 1991; 13: 445-7.

8. Snook JA, de Silva HJ, Jewell DP. The association of autoinmune disorders with inflammatory bowel disease. Q J Med 1989; 72: 835-40.

9. Giannadaki E, Potamianos S, Roussomoustakaki M, Kyriakou D, Fragkiadakis N, Manousos ON. Autoimmune menolytic anemia and positive Coombs test associated with ulcertive colitis. Am J Gastroenterol 1997; 92: 1872-4.

10. Bell DW, Urban E, Sears DA, Walder AI, Ostrower VS. Ulcerative colitis in the hemolytic anemia. South Med J 1981; 74: 359-61.

11. Scully RE, Mark EJ, McNeely WF, Ebeling SH, Ellender SM. Case records of Massachusetts General Hospital 2000; 342: 722-8.

12. Arrizabalaga J, Zulaica D, Clavé E, Iribarren JA, Martín L. Anemia hemolítica autoinmune como complicacióon de neumonia por Mycoplasma pneumoniae. An Med Interna (Madrid) 1986; 3: 136-7.
13. Espinosa JL, Muñoz JA. Anemia hemolítica autoinmune y cirrosis hepática posthepatitis C. An Med Interna (Madrid) 1997; 11: 583-4.

14. Lorber y Balint JA, Hammack WJ, Patton TB. Autoimmune haemolytic anaemia complicating ulcerative colitis. Gastroenterology 1972; 42: 740 .

15. Shashaty GG, Rath CE, Britt EJ. Autoimmune haemolytic anaemia associated with ulcerative colitis. Am J Hematol 1977; 3: 199-208.

16. Yates P, Macht LM, Williams NA, Elson CJ. Red cell autoantibody production by colonic mononuclear cells from a patient with ulcerative colitis and autoimmune haemolytic anaemia. Br J Haemotol 1992; 82: 753-6.

17. Fong S, Fudenberg H, Perlmann P. Ulcerative colitis with anti-erythrocyte antibodies. Vox Sang 1963; 8: 668-79.

18. Basista MH, Roe DC. A case presentation of hemolytic anemia in ulcerative colitis and review of the literature. Am J Gastroenterol 1986; 81: 990-2. 